\title{
Biochemical passivation of metal surfaces for sensor application: reactive annealing of polycrystalline gold films in hydrogen sulfide atmosphere
}

\author{
B. A. Snopok*, K. V. Kostyukevych, G.V. Beketov, S.A. Zinio, Yu. M. Shirshov, E.F. Venger \\ Institute of Semiconductor Physics NASU, 45, prospect Nauki, 03028, Kyiv, Ukraine \\ * B.A.S.: Email: snopok@isp.kiev.ua; Telephone: +(380) (44) 26556 26; Phone (Voice\&Fax): +380 (44) 2651827. \\ S.V. Verevka \\ Palladin Institute of Biochemistry, NAS of Ukraine, 9, ul. Leontovicha, 03030, Kyiv, Ukraine
}

\begin{abstract}
The formation of $\mathrm{Au}_{x} \mathrm{~S}_{y}$ interfacial layer by reactive annealing of gold films in $\mathrm{H}_{2} \mathrm{~S}$ atmosphere is investigated. This seems to be a technologically favorable technique for the large-scale and low-cost fabrication of nondestructive immobilization support for biological molecules. Formation of phases with different chemical functionality and surface topography as a function of reaction time was studied using Atomic Force Microscopy (AFM), Surface Plasmon Resonance (SPR) measurements and biomolecular interaction analysis (trypsin - Soybean Trypsin Inhibitor (STI) reaction). The results obtained confirm the classical two-step model for the sulfide phase formation during reactive annealing. This includes an intermediate formation of a dispersed phase of sulfur followed by its reconstruction to a close-packed sulfide layer. Adsorption of proteins onto a certain sulfide layer passes with retention of a native state of adsorbed molecules. The proposed strategy for formation of biochemical structures (gold/sulfide/proteins) on the surface of physical transducers opens a new way for design and development of novel artificial smart sensing systems. They not only maintain optimal functioning of bioreceptors but also are responsive to their environment.
\end{abstract}

Keywords: polycrystalline gold films, sulfide layers, biosensors, enzymes, soybean trypsin inhibitor, reactive annealing, smart sensing system, Atomic Force Microscopy, Surface Plasmon Resonance.

Paper received 06.01.00; revised manuscript received 10.01.00; accepted for publication 10.01.00.

\section{Introduction}

Much success achieved during investigations of biochemical processes at the molecular level have become possible owing, to a large extent, to the sensor instrument engineering that is actively developing [1]. Various (electrical, thermal, mechanical, optical, etc.) physico-chemical transducers are used in sensor systems. The most promising of them seem to be those based on optoelectronic sensing elements [2]. To illustrate, the systems using surface plasmon resonance (SPR) offer a number of undeniable advantages that are inherent in optical sensor systems [3]. Indeed, using this direct, nondestructive and quantitative technique one can work in situ without additional fluorescent or other tracers [4]. Besides, it is possi- ble to monitor the molecular recognition processes in real time and spend only small-volume samples per single analysis procedure. All mentioned above enables one to obtain additional reliable information on kinetics of processes involving molecular biosystems of high sensitivity (the smallest detectable protein amount is about $\left.0.01 \mathrm{ng} / \mathrm{mm}^{2}[5]\right)$.

Despite the fact that optical transducers where SPR is used possess the above advantages, the following two main requirements substantially retard wide use of such systems when analyzing various biochemical processes occurring at the molecular level. First, to provide the highest responsivity the immobilization layer thickness has to be substantially less than the penetration depth for an evanescent wave (i.e., below 100-200 $\mathrm{nm}$ for radiation from the visible spectral region). Second, adsorption of

\footnotetext{
* To whom correspondence should be addressed.
} 
biomolecules onto the transducer surface has not lead to loss in their functional capabilities.

The restrictions discussed above dictate a need for quest of transition layers that should reduce a destructive effect of metal surface. Besides, the sensing surface of a transducer has to provide optimum conditions for receptor centers to function, as well as retention of high stability and manufacturing compatibility with techniques used to fabricate sensing elements. The most widespread solutions of the above problem are obtaining of (i) transition layers using functionalized mercaptans that form (by selfassembling from a solution) highly ordered stable monomolecular organic coatings on the noble metal surfaces $[6,7]$ and (ii) polysaccharide gel-dextrans $[8,9]$ that form a spatial immobilization matrix on metal surfaces.

Here we propose a simple and handy technique to form layers that provide optimum conditions for functioning of immobilized receptor centers of various natures. Our technique is based on formation of a buffer native layer of proteins on the surface of two-dimensional (2D) monomolecular layer of gold sulfide.

\section{Sensing layers for transducer-based systems}

Since an intimate contact between the sensing biomolecules and transducer is essential for biosensor overall functioning, preparation of a support surface is an important step in the biosensor fabrication $[10,11]$. An analysis of the achievements in bio- and chemical sensors production makes it possible to state some general requirements that should be inherent in sensing elements of the transducerbased sensors:

- immobilization milieu similar to the natural state: conditions at the surface should maintain, or even increase, receptor stability and functioning;

- strong receptor-membrane binding, preferably via covalent coupling;

- directional immobilization: to increase active fraction of receptors, their active sites should face outwards from the electrode surface;

- arrangement of receptor centers: optimal packing density of receptor centers at the interface should be specific for high binding activity;

- negligible nonspecific binding: to achieve high sensitivity and specificity, nonspecific binding at both receptor and membrane should be suppressed;

- controlled immobilization: a mechanism of film formation should preferably be based on self-limiting/ self-assembled approaches;

- «physically» inert support: a film should permit the transducing mechanism to function satisfactorily;

- low-cost technology.

Unfortunately, attempts to design sensing elements that meet these standards were not successful to date, despite the fact that various procedures of immobilization (e.g., adsorption on insoluble matrices [12], entrapment in polymers [13], crosslinking by a multifunctional reagents [14], covalent binding onto a membrane [15], etc.) and types of support (such as dextran matrix [8,9], lipid monolayers [16], biotin-streptavidin systems [17], etc.) have been proposed. In this case sensors based on optoelectronic transducers that are sensitive to the refractive index changes near the transducer surface (caused by the nature, concentration or spatial arrangement of guest molecules [18]) should meet strict requirements, such as small thickness, possibility to be fabricated at the surface of small electrodes, stability in liquid phase, «plane» surface, etc.

When an immobilization layer is formed using biologically active molecules, then the requirement that an interlayer thickness must be minimum becomes decisive. On this basis the soybean trypsin inhibitor (STI) has been chosen to serve as a model system in formation of such a layer. STI is a protein whose relative molecular mass is $21500 \mathrm{Da}$. It involves a polypeptide chain that contains 181 amino groups [19]. This simple protein has a rigidly fixed spatial structure that is favored by two intramolecular disulfide bonds [20]. The structure is tolerant to various denaturation actions (i.e., $\mathrm{pH}$ changing, treatment in concentrated carbamide or guanidine chloride solutions). Special precautions are needed, however, to prevent denaturation of this protein at metal (in particular, gold) surfaces.

\section{Chemical modification of the metal surface of an optoelectronic transducer}

Adsorption of molecular biosystems that are present in living organisms (i.e., enzymes, immunoglobulins, etc.) onto metal surfaces predominantly changes native conformation protein molecules. It is accompanied with both considerable changes in the physico-chemical properties of a protein and complete loss of biological activity $[21,22]$. There is an abundance of experimental evidences that protein secondary and ternary structures unfold at metal surfaces. However, up to now there exists no adequate notion of the physico-chemical processes occurring, and the main factors that determine denaturation have not been clarified yet [23].

For many globular proteins their complete spatial structure is equivalent to their ternary structure. The latter is a relatively compact system where the secondary structure elements (canonical polypeptide chain conformations that are usually stabilized with hydrogen bonds, such as $\alpha$-helices, $\beta$-structures, etc.) are, in many cases, linked to both each other and the disordered structure sections through disulfide bonds [24]. According to this, one may assume that changes in native conformation may be, in particular, due to breaking of intramolecular disulfide bonds. Indeed, such reagents as $\beta$-mercaptoethanol and dithiotreitol (that restore disulfide bonds) usually promote protein denaturation. The same result may be obtained through the interaction between an intramolecular disulfide bond and substrate metal (in particular, gold) atoms. Numerous experiments in forma- 


\section{B. A. Snopok et al.: Biochemical passivation of metal surfaces...}

tion of the self-assembled monolayers of aliphatic mercaptans from thiol solutions, as well as the corresponding disulfides on gold surface, unambiguously are indicative of breaking disulfide bonds at the metal surface (in the second case) and formation of monolayers that are structurally and chemically equivalent (in both cases) $[25,26]$. Thus, if at the sensing element surface the conditions exist that prevent disulfide bond breaking, then this may provide a retention of the native conformation for biological molecules.

$\operatorname{Metal}(\mathrm{Au}, \mathrm{Ag}, \mathrm{Cu}, \mathrm{Fe}$, etc.) sulfides are known to be both kinetically and thermodynamically stable compounds, reactivity of which, as to the chemical groups that are characteristic of biological molecules is negligible. Besides, orderly arranged disulfide monomolecular coatings could be obtained using a simple and technologically easy treatment of the corresponding metal surfaces in the hydrogen sulfide atmosphere. Comprehensive investigations of interaction between hydrogen sulfide and variously oriented surfaces of metal single crystals have been performed in wide pressure $\left(10^{-2}-10 \mathrm{~Pa}\right)$ and temperature $\left(100-350^{\circ} \mathrm{C}\right)$ ranges for $\mathrm{Cu}-\mathrm{S}$ [27], Ni-S [28], Ag-S [29], Fe-S [30] and Au-S [31] systems in the hydrogen sulfide-hydrogen atmosphere. Reversible chemisorption, low-energy electron diffraction and Auger electron spectroscopy have been used in combination with sulfur $\mathrm{S}^{35}$ isotope. This made it possible to (i) investigate thermodynamic aspects of processes occurring in the systems studied; (ii) exert control over the surface chemical composition; (iii) determine both growth mechanism and structure for surface sulfide, and (iv) deduce an exact number of adsorbed sulfur atoms per unit area.

For differently oriented gold single crystals the mechanism of interaction with hydrogen sulfide vapors is given in detail in [32]. It was shown that the island phase adsorption previously occurs, with sulfur atoms localized at places of highest metal coordination. When a critical coating with the first island phase is reached, then coalescence of individual assemblies leads to formation of rather big areas of continuous sulfide. Further increase in sulfur amount on the surface results in formation of close-packed coatings of the highest filling. Formation of a new surface phase is accompanied with metal surface reconstruction due to the growth of a $2 D$ gold sulfide layer. No atoms other than sulfur have been detected in the surface coating obtained. For all the crystal faces the coating formation is not an ordinary epitaxial growth on the substrate. It induces appearance of new faces as a result of reconstruction. Whatever the face orientation and conditions of adsorption process, the same amount of sulfur per unit area under saturation $\left(\approx 0.39 \mathrm{ng} / \mathrm{mm}^{2}\right)$ was observed. This value characterizes formation of a complete monolayer. $2 D$ adsorbed sulfide remains stable in a vacuum at temperatures up to $250^{\circ} \mathrm{C}$. Such a low sublimation temperature serves as an indication that the surface compound is $\mathrm{Au}_{\mathrm{x}} \mathrm{S}_{\mathrm{y}}$. The minimum spacing between sulfur atoms in the surface monolayer obtained is $d(\mathrm{~S}-\mathrm{S})=$ $0.364 \mathrm{~nm}$; heat of adsorption for sulfur is $86 \mathrm{kcal} / \mathrm{mole}$.

\section{Materials and methods}

Both trypsin $\left(\mathrm{M}_{\mathrm{r}} \sim 22500 \mathrm{Da}\right)$ and Soybean Trypsin Inhibitor $\left(M_{r} \sim 21500 \mathrm{Da}\right)$ have been purchased from REANAL (Hungary) and used as received. Fresh solutions of concentration $200 \mu \mathrm{g} / \mathrm{ml}$ in a buffer were prepared just before starting experiment. All the subsequent reaction steps were performed in Phosphate buffered Saline (PBS), $\mathrm{pH}=7.2$, at an ambience temperature of $20 \pm 2{ }^{\circ} \mathrm{C}$. All the chemical reagents used by us were «pure» purity class.

Hydrogen sulfide was prepared in a laboratory by adding diluted hydrochloric acid to iron sulfide [33]. The samples were treated in saturated $\mathrm{H}_{2} \mathrm{~S}$ vapor at room temperature and atmospheric pressure during predetermined time intervals $(5,10,15,20$ and $25 \mathrm{~h})$. Such a procedure has been chosen proceeding from the following requirement: the manufacturing technology for biosensor sensing elements must be simple. It provided high reproducibility of fabricated samples.

The supports were glass plates (measured $38 \times 18 \times 1 \mathrm{~mm}$ ) or trapezoid prisms (measured $18 \times 12 \times 6 \mathrm{~mm}$, an inclination angle of $68^{\circ}$ ) with the refractive index of 1.515. To obtain the samples, first an adhesive layer of $\mathrm{Cr}$ (about $5 \mathrm{~nm}$ thick) and then an Au layer ( $45 \mathrm{~nm}$ thick) were sputtered onto the upper side of the glass plate/prism. During the deposition the plate/prism was kept at $22^{\circ} \mathrm{C}$ with subsequent holding (for three hour) in a vacuum (pressure of $5 \times 10^{-4} \mathrm{~Pa}$ ) before being taken out of the evaporation equipment (VUP-5M). The obtained plates/prisms were used for hydrogen sulfide treatment without any additional cleaning procedures before the experiment.

AFM imaging was performed using a commercial Nanoscope IIIa (Digital Instrument, Santa-Barbara) equipped with an $80 \mu \mathrm{m}$ scanner. The unique plates, both untreated and treated with $\mathrm{H}_{2} \mathrm{~S}$, were used for the AFM analysis. The scans were performed in the tapping force mode using commercially available AFM tips made of silicon nitride. The scan frequency was about $0.3 \mathrm{~Hz}$.

The effect of $\mathrm{H}_{2} \mathrm{~S}$ treatment on the SPR response was investigated using a universal homemade installation whereas the adsorption processes were investigated with the SPR instrument BioHelper PLASMON-003 (Institute of Semiconductor Physics NASU, Kiev, Ukraine) [34]. In both SPR instruments the Kretschmann configuration was employed. A light beam from the He-Ne laser (wavelength $\lambda=632.8 \mathrm{~nm}$ ) was directed onto the bottom face of a glass prism. Its top side was coated with a thin gold film, thus providing total internal reflection at the glass/metal interface. The reflectivity of a laser beam in this system was measured as a function of an angle of incidence. At a certain value of the angle of incidence coupling between photons from the laser beam and electron gas at the outer metal interface causes excitation of surface plasmons, thus leading to a reduction in the intensity of reflected light. The angle at which this excitation occurs depends on the dielectric properties of both the metal film and layers above the sensor surface. The 
resulting response (denoted as SPR reflectivity curve) has several distinct features and can be quickly characterized by the position of the minimum (denoted as SPR angle $\left.\theta_{S P R}\right)$, reflection value at the minimum $\left(R_{\mathrm{SPR}}\right)$ and half-width $\left(R_{1 / 2}\right)$. Of particular importance for sensor application of SPR phenomena is adsorption of molecules on the chip surface. It causes the SPR angle variation, and hence the $\theta_{\text {SPR }}$ shift may serve to detect processes occurring on the surface in real time and without any additional labels.

The experimental cell was formed of silicone rubber covered by the Teflon holder. It was mounted immediately onto the sensor chip surface. The cell was of circular section (cross-section of about $25 \mathrm{~mm}^{2}$ ) normally to the prism surface. Its height was above $1 \mathrm{~mm}$ and volume of about $25 \mu 1$.

Analysis of kinetic curves was made in accordance with the classical Langmuir kinetic theory. For direct analysis of SPR data the following expressions were used $[35,36]$ :

$$
\Gamma=\Gamma_{\max }\left[1-\exp \left(-k_{e f f} t\right)\right]
$$

or

$$
\Delta \theta_{S P R}=\theta_{\text {sat }}\left[1-\exp \left(-k_{\text {eff }} t\right)\right] .
$$

Here $\Gamma$ is the bound analyte coverage, $\Gamma_{\max }$ is the maximum coverage, $\theta_{\text {sat }}$ is the plateau value of the SPR angle shift $\Delta \theta_{S P R}, k_{\text {eff }}$ is the rate constant, $t$ is time. The analyte dissociation can be studied by replacing the analyte solution by buffer and is described by the following equation:

$$
\Gamma=\Gamma_{b} \exp \left[-k_{\text {diss }}\left(t-t_{b}\right)\right] .
$$

Here $\Gamma_{b}$ is the surface coverage at the point of time $t_{b}$ when the analyte solution is replaced with the buffer.

\section{Results and discussion}

Let us first discuss the AFM characterization of the topography peculiarities for gold surface treated with $\mathrm{H}_{2} \mathrm{~S}$. Then the SPR investigation of both initial gold films and those reconstructed during formation of a sulfide layer will be described in detail. And, finally, let us discuss possible mechanisms of STI adsorption onto initial and treated with $\mathrm{H}_{2} \mathrm{~S}$ films and its subsequent interaction with trypsin.

\subsection{Topography of initial and treated with $\mathrm{H}_{2} \mathrm{~S}$ gold-coated prism surfaces}

The gold-coated plates were initially imaged by AFM to characterize the native surface. The AFM pattern (see Fig. 1) shows that the surface is not atomically smooth. The gold grain diameter ranges from 20 to $30 \mathrm{~nm}$. The surface roughness shows a maximum height variation of $2.5 \mathrm{~nm}$ for a $500 \mathrm{~nm}$ scan, with the root-mean-square (rms) surface roughness about $0.84 \mathrm{~nm}$.
Structural changes for the initial samples exposed to saturated hydrogen sulfide vapors begin at the «trenches» (see Fig. 1). Their curvature decreases, presumably, due to the preferential sulfur adsorption in these regions. When the exposition time is increased up to $10 \mathrm{~h}$, then the topography of the whole crystallite surface changes. The general features of all the above surface structures are (i) presence of a small-scale roughness over the whole surface of individual crystallites and (ii) absence of those surface structures whose mean size exceeds that typical for the initial gold surface.

An increase of exposition time up to $15 \mathrm{~h}$ essentially changes the above surface pattern. First of all, a substantial decrease of the small-scale roughness occurs. The surface profile may be presented as a set of monotonously smooth curves with a pronounced (up to about $50 \%$ ) contribution from quasiflat portions. Such a pattern is an indication at a correlated occurrence of the surface reconstruction processes, at least on a scale of a single or few crystallites. Further (up to 20 and $25 \mathrm{~h}$ ) growth of exposition time leads to further (down to $\sim 0.5-0.6 \mathrm{~nm}$ ) decrease of the rms roughness and an increase (up to macroscopic sizes) in mean sizes of the regions where correlated reconstruction occurred. It should be noted that, as can be seen from the film surface patterns given in Fig. 1, the arranged structures that can be observed after treatment with hydrogen sulfide vapor for $20 \mathrm{~h}$ undergo transfer to macroscopically uniform regions sizes.

Therefore our investigations of topography performed for thin gold films, both initial and treated with saturated $\mathrm{H}_{2} \mathrm{~S}$ vapor for different time intervals, enabled us to conclude that, during the surface sulfide layer growth, two phases are successively formed, namely:

- an early, or island, phase that is characterized by a presence of a small-scale roughness and uncorrelated growth of individual areas;

- a coalescent phase resulting from coalescence of individual assemblies and cooperative reconstruction of large surface areas.

It should be noted that this mechanism of the sulfide layer formation on thin polycrystalline polyoriented films agrees well with the previously discussed sulfide layer formation on different faces of single-crystalline metals.

\subsection{Hydrogen sulfide interaction with a gold- coated plate: SPR analysis}

The structural peculiarities of the sulfide layer formation on a polycrystalline gold surface that have been discussed above are to affect also the generation of surface polaritons in such systems. Indeed, the conditions for generation of a charge density wave that is localized near the surface substantially depend on surface topography, since the electric field of a plasmon is localized on the metal side within a distance of several lattice periods [37]. Therefore both the surface roughness and nonuniformity of the dielectric parameters of a transition layer on frequently occasions make an uncontrollable contribution 

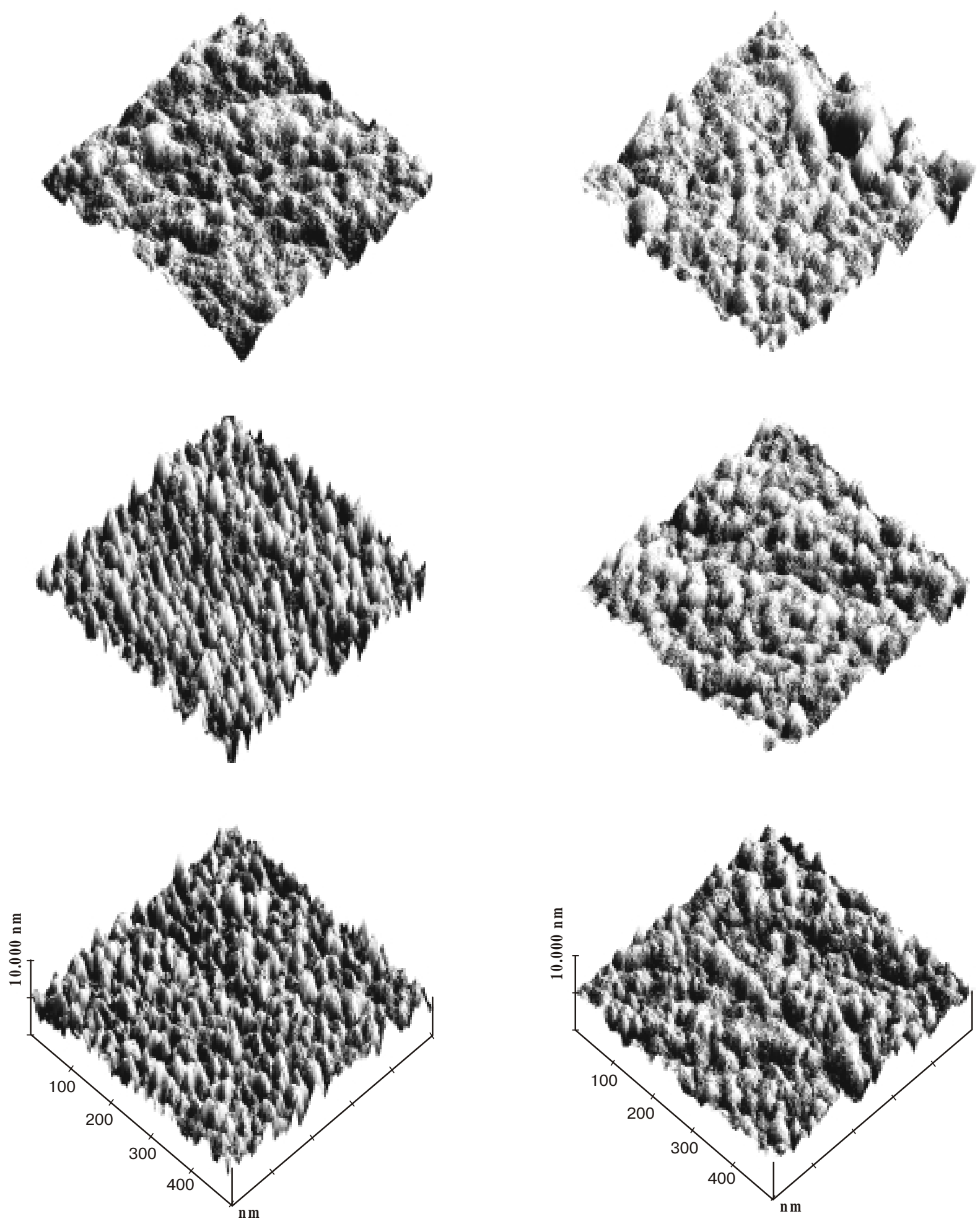

Fig. 1. An atomic force microscope image (500 nm square scan) of the untreated gold surface (bottom left) and AFM images of treated by $\mathrm{H}_{2} \mathrm{~S}$ gold films during different time: 5 (middle left), 10 (top left), 15 (bottom right), 20 (middle right) and 25 (top right) hours. 


\section{B. A. Snopok et al.: Biochemical passivation of metal surfaces...}

to the effective values of optical constants at the interface. Taking into account the above features of the processes occurring at the surface, one may conclude that treatment in the hydrogen sulfide vapor may lead to the following two effects: (i) formation of a new compound, namely, gold sulfide $\mathrm{Au}_{x} \mathrm{~S}_{y}$ at the film surface, and (ii) reconstruction of the near-surface metal layer. Both effects have to result in substantial changes in the observed SPR curves.

The resonant SPR curves taken out of doors for both initial samples and those exposed to the hydrogen sulfide vapor for 5, 10, 15, 20 and $25 \mathrm{~h}$ are presented in Fig.2. One can see that during the first ten hours of the above treatment the SPR curve is shifted toward bigger angles and widened; besides, the $R_{\mathrm{SPR}}$ value grows. Such changes are indicative of nonuniform insulating layer formation at the surface.

When the treatment duration is increased over $15 \mathrm{~h}$, then the SPR curve shifts toward smaller angles, $R_{\mathrm{SPR}}$ grows and the halfwidth, $R_{1 / 2}$, drops. Besides, a considerable drift decrease for the SPR curve minimum in water solutions (that is characteristic of the samples exposed to treatment for over $15 \mathrm{~h}$ ) indicates that formation of a continuous unsolvable coating (a sulfide layer) takes place. This process is accompanied with reconstruction of the gold surface. The above coating provides strong chemical passivation of the polycrystalline polyoriented gold films.

Thus a comparison between the SPR curves taken for the initial samples and those exposed to hydrogen sulfide vapor for different time intervals enables one to conclude that:

- initial phase formation may be adequately described as formation of nonuniformly adsorbed sulfur associates;

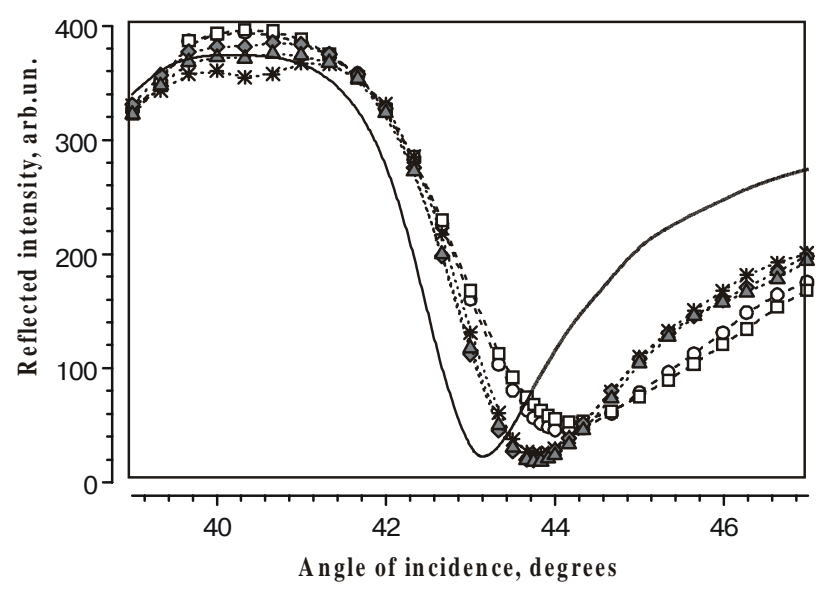

Fig. 2. The SPR reflectivity curves for a clean gold surface (solid line) and the same surface after treating during different time in $\mathrm{H}_{2} \mathrm{~S}$ atmosphere: 5 ( ), $10(\mathrm{O}), 15(\diamond), 20(\mathbf{\Delta})$ and $25(\boldsymbol{*})$ hours.
- growth of the second phase is due to the gold surface reconstruction resulting from the correlated growth of sulfide layer on a macroscopic scale.

\subsection{Interaction between trypsin and adsorbed STI}

The highly specific interactions that are characteristic of the molecular biosystems involved in the immune response of living organisms (antigen - antibody reaction) are well known. They are widely used to form sensing layers in various biosensor systems. At the same time another class of biologically active molecules, namely, enzymes and their inhibitors until recently has not found practical application as molecular components of sensing elements in optical biosensors.

The physiological functions of proteins-inhibitors stem from their ability to reversibly bind enzymes, i.e., take part in regulation of enzyme activity. In this way they prevent premature decay of spare proteins, take part in the metabolism regulation in plants, neutralize enzymes of phytopathogenic microorganisms, etc. [38]. To illustrate, trypsin (belonging to the class of proteolytic enzymes) plays a key role in metabolic processes occurring in living organisms. It is involved in both decay and formation of physiologically important proteins and peptides [39]. STI (the Kunitz inhibitor that is obtained from the soybeans $[40,41])$ is a natural trypsin inhibitor. It possesses high specificity to trypsin.

The basis for the reaction between proteins-inhibitors and enzymes is formation of steady complexes that contain molecules of both inhibitor and enzyme. High degree of complementarity for interacting surfaces of two protein molecules is possible due to a great number of intermolecular interactions. Along with hydrogen bonds, a lot of hydrophobic contacts appear. They contribute significantly to the formation of stable complexes. All these facts enable one to conclude that possibility of the enzyme-inhibitor complex formation is determined, to a great extent, by the stability of the protein secondary structure. Therefore, one may reason that efficiency of such a highly specific interaction serves as an evidence that the native conformation of molecules taking part in the reaction is retained.

In order to fabricate a STI-based biofilm on the initial gold surface and that treated with $\mathrm{H}_{2} \mathrm{~S}$ typical adsorption experiments were performed. The PBS buffer was allowed to flow through the cell and then abruptly was changed for the STI solution with concentration of $200 \mu \mathrm{g} / \mathrm{ml}$. After the sensor response reached an equilibrium state without flow, PBS, in its turn, was replaced with the STI solution. Then this procedure was repeated with injection of a portion of trypsin solution in PBS with concentration of $200 \mu \mathrm{g} / \mathrm{ml}$. The results of these experiments are shown in Figs 3-4. One can see that the SPR angle increases with STI adsorption onto the initial gold surface (Fig. 3) are flattens out, giving rise to an SPR angle shift of $c a .1450 \pm 100^{\prime \prime}$ within a period of about $1000 \mathrm{~s}$.

SQO, 3(1), 2000 


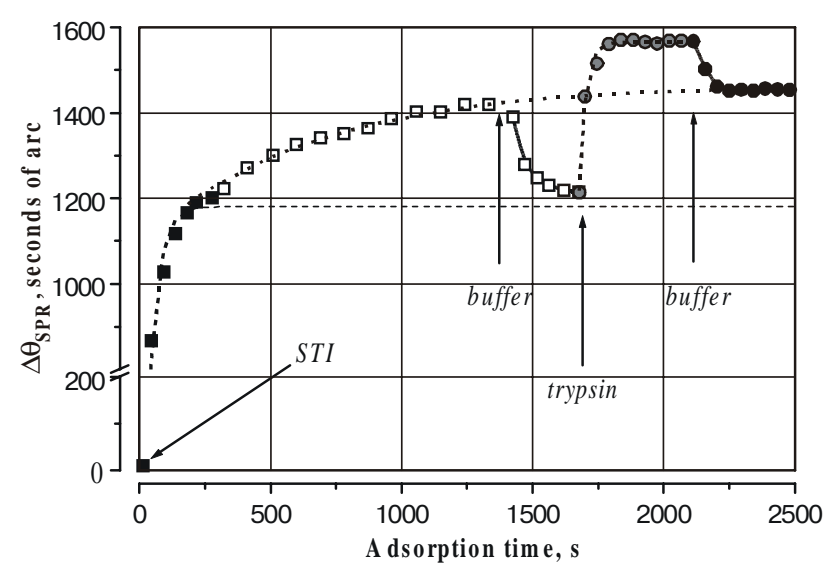

Fig. 3. Adsorption of STI onto unmodified gold surface and trypsin interaction with adsorbed STI layer. Nonlinear regression analysis (applying Equation 1) gives the following solution: for STI onto the gold surface - $\Theta_{\text {sat }}^{1}=1180 ", k_{\text {eff }}^{1}=0.027 \mathrm{~s}^{-1}$ (for primary layer - dash line) and $\Theta_{\text {sat }}^{2}=302 ", k_{\text {eff }}^{2}=0.0016 \mathrm{~s}^{-1}$ (for secondary layer - dotted line); for reaction of trypsin with adsorbed STI layer - $\Theta_{\text {sat }}=400^{\prime \prime}$, $k_{\text {eff }}=0.043 \mathrm{~s}^{-1}$ (dash line).

For adsorbed proteins their surface concentration can be calculated from the SPR data in accordance with [35]. If one takes for the refractive index increment the average value of $0.188 \mathrm{~cm}^{3} \mathrm{~g}^{-1}$ [42] for both STI and trypsin, then the adsorbed amount of STI on the initial gold surface gives experimental value of about $1.5 \mathrm{ng} / \mathrm{mm}^{2}$. This is essentially lower than the limiting value for full surface coverage (about $5 \mathrm{ng} / \mathrm{mm}^{2}$ ). Thus it is possible to suggest that protein folding is inherent for STI adsorption onto the initial gold surface. Indeed, the interaction between trypsin and the adsorbed STI layers show a very small response (about $250^{\prime \prime}$ or $0.25 \mathrm{ng} / \mathrm{mm}^{2}$ ). This fact may be interpreted as resulting from imperfection of the native molecular state at the surface. In this connection a complicated form of the kinetic curve for STI adsorption onto the native gold surface is noteworthy.

In fact, this curve cannot be described with a simple first-order Langmuir model. However, the data are consistent with a multistage formation mechanism when a fast formation stage is followed by a slow formation one. Indeed, Fig. 3 indicates that the kinetic curves are well described by a sequence of distinct first-order kinetic steps. We assume that the curve shape observed by us shows that the adsorption process has two stages with different both rate constants and strengths of molecules coupling with the surface. It should be particularly emphasized that each stage is described very well with a simple monomolecular one-to-one interaction model.

A model that involves the existence of a «loosely» adsorbed layer on top of the primary monolayer can explain the experimental data [34]. The latter layer that is immediately adjacent to the surface contains irreversibly coupled protein molecules. Thus it seems reasonable to suggest that a simple «Langmuir-like» adsorption mode when the protein-gold interaction (a surfaceinduced folding $[43,44]$ of STI that occurs concurrently with adsorption) dominates is changed for the native protein from solution - folded surface coupled protein interactions at the second layer formation. It should be particularly emphasized that the contribution from the protein - protein physisorption to the total biosensor response can be essential only after formation of the most part of the first layer. According to this assumption, we have used an approach of time - division binding equilibrium described by a simple Langmuir binding model.
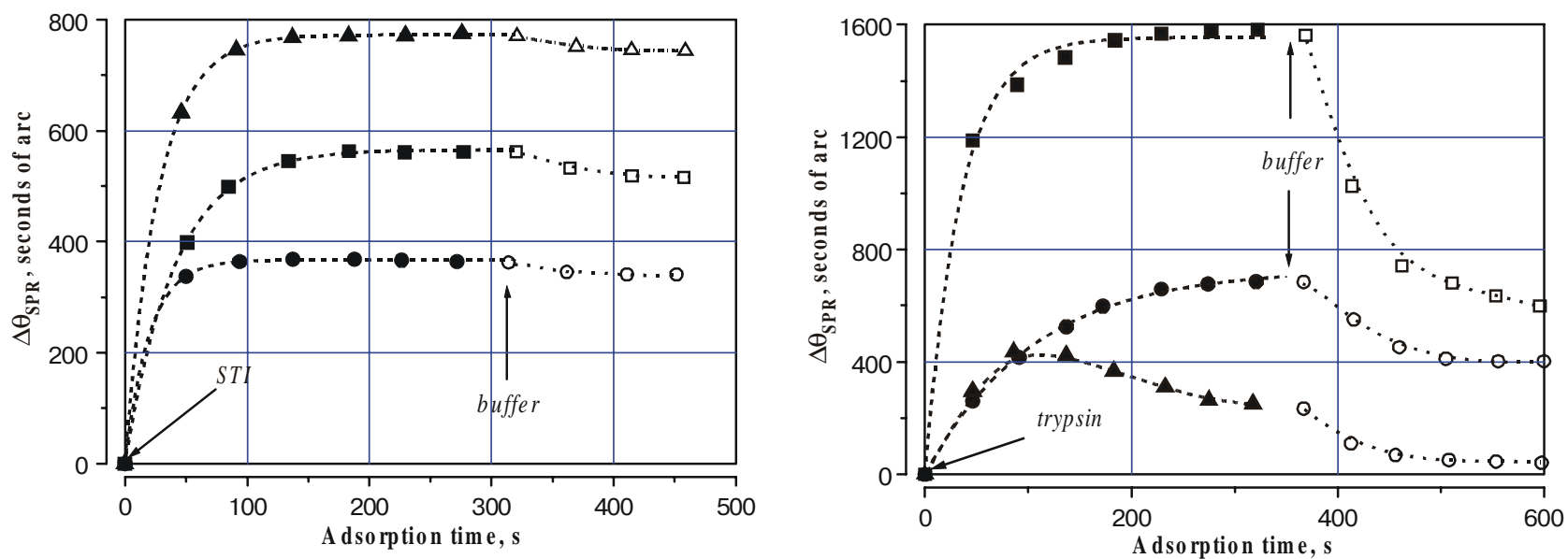

Fig. 4. Adsorption of STI ( $200 \mu \mathrm{g} / \mathrm{ml}$ in PBS) onto gold films treated during different times in $\mathrm{H}_{2} \mathrm{~S}$ atmosphere (left) and interaction of adsorbed STI layers with trypsin $(200 \mu \mathrm{g} / \mathrm{ml}$ in PBS) (right): $5(\mathbf{\square}, \mathbf{\square}), 15(\mathbf{O}, \bullet)$ and $25(\Delta, \mathbf{\Delta})$ hours. Nonlinear regression analysis (applying Equation 1 - dash line) gives the following solution: for STI $\Theta_{\text {sat }}=770$ ", $k_{\text {eff }}=0.037 \mathrm{~s}^{-1}$ (for $5 \mathrm{~h}$ ), $\Theta_{\text {sat }}=565$ ", $k_{\text {eff }}=0.0244 \mathrm{~s}^{-1}$ (for $15 \mathrm{~h}$ ); $\Theta_{\text {sat }}=367 ", k_{\text {eff }}=0.0003 \mathrm{~s}^{-1}$ (for $25 \mathrm{~h}$ ) and for reaction of trypsin with adsorbed STI layer: $\Theta_{\text {sat }}=1555$ ", $k_{\text {eff }}=0.029 \mathrm{~s}^{-1}$ (for $15 \mathrm{~h}$ ); $\Theta_{\text {sat }}=$ 731 ", $k_{\text {eff }}=0.0095 \mathrm{~s}^{-1}$ (for $25 \mathrm{~h}$ ). 
At the same time adsorption of trypsin onto the STI-modified gold surface is well described by a simple monomolecular one-to-one interaction model. It is necessary to stress that total amount of adsorbed (irreversible and «loosly») STI onto unmodified gold surface is about the same as one for summary contribution from strong coupled STI fraction and specifically adsorbed part of trypsin molecules.

For the samples treated with hydrogen sulfide for different time intervals the interaction with STI differs substantially from the one discussed above for the unmodified gold surface (Fig. 4a). First of all, the maximum amount of adsorbed STI is much less than that in the case of the unmodified sample and depends on the duration of treatment with $\mathrm{H}_{2} \mathrm{~S}$ vapor. The amount of irreversible adsorbed protein decreases with time for the samples obtained during the first phase formation $(0.8$ and $0.6 \mathrm{ng} /$ $\mathrm{mm}^{2}$ for 5 and $10 \mathrm{~h}$ of treatment, respectively) and remains the same ( $c a .0 .4-0.5 \mathrm{ng} / \mathrm{mm}^{2}$ ) during further treatment. The kinetic curves indicate at a one-stage character of the adsorbed STI layer formation. It is necessary to stress that no extra loosely bound molecules have been removed from the sensor surface during washing in pure PBS. Interaction between trypsin and the samples obtained on the layers modified with hydrogen sulfide during the first phase stage indicates that a contribution from the specific interaction is small: the amount of bound trypsin in this case is 10-20\% the adsorbed STI amount.

Qualitative distinctions exist between the pattern observed for the samples that have been obtained during the second phase formation and that discussed above. An approximation of the kinetic curve for STI adsorption for the samples exposed to hydrogen sulfide for more than $15 \mathrm{~h}$ evidences that in this case the adsorption process also obeys the classical Langmuir model $[45,46]$. This fact means, in particular, that the modified gold surface behaves (in respect to protein adsorption) as energetically uniform, with a constant amount of noninteracting adsorption sites whose characteristics are time-independent. In this case the amount of specifically bound trypsin indicates at formation of a trypsin-STI complex whose components are in the ratio of 1:1 (Fig.5). And essential amount of a loosely bound nonspecific component is observed that is removed by washing in a buffer. It is necessary to stress that treatment of complexes STI-trypsin by $\mathrm{pH}=2.2$ solutions (Fig.6) shows that this reaction is specific and reversible.

All the above facts make it possible to conclude that the STI layer in the native conformation on the modified gold surface (that is obtained during the second stage) is not continuous. In this case the interaction between trypsin and the adsorbed STI layer can be adequately described within the classical Langmuir theory. It is of particular interest that the total amount of adsorbed trypsin (both specifically and loosely bound) may be described within the same adsorption equilibrium. This evidences that the whole surface (covered with STI, as well as STI-free) is characterized by the same trypsin adsorption ability.

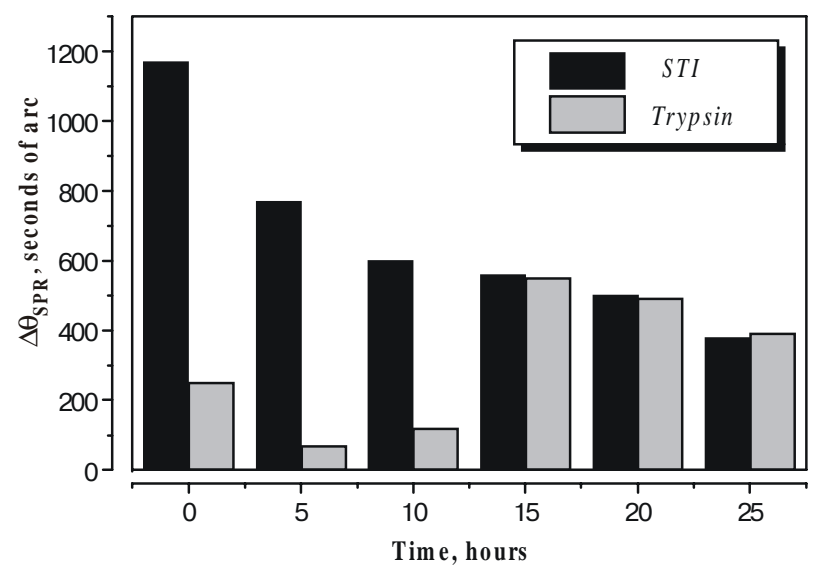

Fig.5. Saturated values of the SPR angle shift for irreversibly adsorbed STI $(200 \mu \mathrm{g} / \mathrm{ml}$ in PBS) onto unmodified and treated surfaces and one for subsequent specific interaction of adsorbed STI layer with trypsin $(200 \mu \mathrm{g} / \mathrm{ml}$ in PBS) versus reaction time of reactive annealing of thin gold films in $\mathrm{H}_{2} \mathrm{~S}$ atmosphere.

It has been noted earlier that the STI layer on gold surface is characterized by a low (about $10 \%$ of the limiting value) degree of monolayer filling. This may result from the following two reasons. The STI isoelectric point is $\approx 4.5$ [47]. So at $\mathrm{pH}=7.2 \mathrm{STI}$ is in the charged form. This can limit the close-packed structure formation. On the other hand, the polycrystalline structure of gold surface may, in its turn, substantially affect the adsorbed STI layer structure. To illustrate, if one takes that STI is a ball whose diameter equals about $2 \mathrm{~nm}$, then the area of adsorption sites in «trenches» of the polycrystalline structure is about $10-15 \%$ of the substrate geometric area. This agrees well with the observed amount of STI bound at the surface.

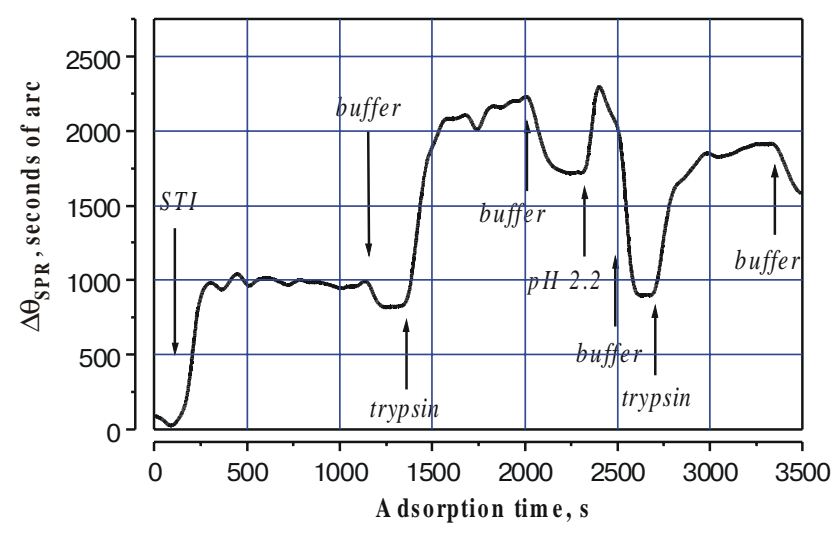

Fig. 6. Influence of washing the STI-trypsin complexes by solutions with low $\mathrm{pH}$ value $(\mathrm{pH} 2.2)$. The reversibility of STI-trypsin reaction indicates that interaction between those molecules has specific character and both STI and trypsin are in native form at the surface. Support is gold film treated in $\mathrm{H}_{2} \mathrm{~S}$ atmosphere during ca.17 hours, both STI and trypsin concentration in PBS is $200 \mu \mathrm{g} / \mathrm{ml}$. 


\section{B. A. Snopok et al.: Biochemical passivation of metal surfaces...}

\section{Concluding remarks}

The most commonly used approach to the sensing layer formation employs either the biomimetic methodology [48] with simplified models for natural products or the biotechnology approaches [49] based on utilization of native biomolecules. However, to get novel sensor systems one has to integrate the major achievements gained in both fields. This would enable to take advantage of high responsivity and specificity inherent in bioreceptors, on the one hand, and both reproducibility and stability of artificial membranes (immobilization support systems), on the other hand.

At the same time an analysis of sensing elements functioning in biological systems makes it possible to identify a specific adaptive character of the measuring process itself. This means that a sensing system adjusts to changes in its ambience to provide optimum functioning (the so-called smart systems). Indeed, most of protein molecules are complex supramolecular systems. They perform several functions that are related to each other. In this case the protein conformation, its charge state, solvate shell composition and a number of functional characteristics depend on both the state and composition of the ambience where it is. According to the above, the development of systems capable not only to keep a receptor biomolecule in its native state but also to provide the optimum conditions for its functioning depending on ambience seems to present an urgent scientific problem.

In this paper we dealt with a strategy to form immobilization layers for such adaptive sensing systems. This strategy is based on formation of a layer of native biomolecules at the transducer surface. Such a layer could serve as a matrix for immobilization of receptor centers of biological nature.

It is known that even the simplest molecular biosystems in living organisms manifest an adaptive character of their behavior during various biochemical processes. Taking this into account, one may expect that receptor systems of different origin, being fixed at the above layer using standard immobilization procedures, will be in optimum conditions that are comparable to those at the biological membrane surfaces [50].

\section{References}

1. A.Brecht, G.Gauglitz, W.Goepel, Sensors in Biomolecular Interaction Analysis and Pharmaceutical Drug Screening in Sensors update 5, H. Baltes, W. Goepel, J. Hesse, Eds. WILEY-VCH, Weinheim (1999).

2. J.J.Ramsden, Optical biosensors // J. Mol. Recognition 10, pp. 109-120 (1997).

3. W.Knoll, Optical characterization of organic thin films and interfaces with evanescent waves // MRS Bulletin 16 (7), pp. 29-39 (1991)

4. Z.Salamon, H.A.Macleod, G.Tollin, Surface plasmon resonance spectroscopy as a tool for investigating the biochemical and biophysical properties of membrane protein systems // Biochimica et Biophysica Acta 1331, pp. 117-152(1997).
5. L.S.Jung, C.T.Campbell, T.M.Chinowsky, M.N.Mar, S.S.Yee, Quantitative interpretation of the response of surface plasmon resonance sensors to adsorbed films // Langmuir 14, pp. 5636-5648 (1998).

6. T.Weib, K.D.Schierbaum, U.T. van Velzen, D.N.Reinhoudt, W.Gopel, Self-assembled monolayers of supramolecular compounds for chemical sensors // Sensors and Actuators B pp. 26-27, 203-207 (1995).

7. M.W.J.Beulen, M.I.Kastenberg, F.C.J.M. van Veggel, D.N.Reinhoudt, Electrochemical stability of self-assembled monolayers on gold // Langmuir 14, $7463-7467$ (1998).

8. B.Liedberg, I.Lundstrom, E.Stenberg, Principles of biosensing with an extended coupling matrix and surface plasmon resonance // Sensors and Actuators B 11, pp. 63-72 (1993).

9. S.Loefas, B. Johnsson A novel Hydrogel Matrix on Gold Surfaces in Surface Plasmon Resonance Sensors for Fast and Efficient Covalent Immobilization of Ligands // J.Chem.Soc., Chem.Commun., 1526-1528 (1990).

10. W.Knoll, L.Angermaier, G.Batz, T.Fritz, S.Fujisawa, T.Furuno, H.-J.Guder, M. Hara, M.Liley, K.Niki, J.Spinke, Supramolecular engineering at functionalized surfaces // Synthetic Metals 61, pp. 5-11 (1993).

11. R.M.Crooks, A.J.Ricco, Neworganic materials suitable for use in chemical sensor // Accounts of Chemical Research 31(5), pp. 219-227 (1998)

12. M. Knichel, P.Heiduschka, W.Beck, G.Jung, W.Goepel, Utilization of a self-assembled peptide monolayer for an impedimetric immunosensor // Sensors and Actuators B 28, pp. 85-94 (1995)

13. K.Arisumi, F.Feng, T.Miyashita, H.Ninomiya, Functionalization of polymer Langmuir-Blodgett films using active ester groups // Langmuir 14, pp. 5555-5558 (1998).

14. W.H.Scouten, J.H.T.Luong, R.S.Brown,IEnzyme or protein immobilization techniques for applications in biosensor design // Tibtech 13, pp. 178-185 (1995).

15. B.Raguse, V.Braach-Maksvytis, B.A.Cornell, L.G.King, P.D.J.Osman, L.Wieczorek, Tethered Lipid Bilayer Membranes: Formation and Ionic Reservoir Characterizatio // Langmuir 14(3), pp. 648-659 (1998).

16. B.Raguse, V.Braach-Maksvytis, B.A.Cornell, L.G.King, P.D.J.Osman, R.J.Pace, L.Wieczorek, Tethered lipid bilayer membranes: formation and ionic reservoir characterization // Langmuir 14, pp. 648-659 (1998).

17. F.-J. Schmitt, L.Haussling, H.Ringsdorf, W.Knoll, Surface plasmon studies of specific recognition reactions at self-assembled monolayers on gold // Thin Solid Films 210-211, pp. 815-817 (1992)

18. S.Spinke, M.Liley, F.J.Schmitt, H.S.Guder, L.Angermaier, W.Knoll, Molecular recognition at self-assembled monolayers: optimization of surface functionalization // J.Chem.Phys. 99, pp. $7012-7019$ (1993)

19. K.Ozawa, Jr. M. Laskowski, The reactive site of trypsin inhibitors // J. Biol. Chem 241, pp. 3955-61 (1966).

20. H.K.Song, S.W.Suh, Kunitz-type soybean trypsin inhibitor revisited: refined structure of its complex with porcine trypsin reveals an insight into the interaction between a homologous inhibitor from Erythrina caffra and tissue-type plasminogen activator // J. Mol. Biol. 275, pp. 347-63 (1998).

21. J.E.Lee and S.S.Saaverda, Molecular orientation in heme protein films adsorbed to hydrophilic and hydrophobic glass surfaces // Langmuir 12, pp. 4025-4032 (1996).

22. Comprehensive Supramolecular Chemistry, J.-M.Lehn, Eds., Pergamon (1996).

23. V.P.Zhdanov, B.Kasemo, Monte Carlo simulations of the kinetics of protein adsorption // Surf. Rev. Lett. 5 (2), pp. 615634 (1998).

24. Yu.A.Ovchinnikov, Bioorganic chemistry, Prosveshchenie, Moskva (1987).

25. Ch.Jung, O.Dannenberger, Y.Xu, M.Buck, M.Grunze, Selfassembled monolayers from organosulfur compounds: a comparison between sulfides, disulfides, and thiols // Langmuir 14, pp. 1103-1107 (1998). 


\section{B. A. Snopok et al.: Biochemical passivation of metal surfaces...}

26. H.Schonherr, H.Ringsdorf, Self-assembled monolayers of symmetrical and mixed alkyl fluroalkyl disulfides on gold 1. Synthesis of disulfides and investigation of monolayer properties // Langmuir 12, pp. 3891-3897 (1996).

27. J.L.Domange, J.Oudar, Structure et conditions de formation de la couche d'adsorption du soufre sur le cuivre // Surf. Sci. 11, pp. 124-142 (1968).

28. M. Perdereau, J.Oudar, Structure, mecanisme de formation et stabilite de la couche d'adsorption du soufre sur le nickel // Surf. Sci. 20, pp. 80-98 (1970).

29. J.Benard, J.Oudar, F.Cabane-Brouty // Surf. Sci. 3, p. 359 (1965).

30. J.Oudar, E.Margot, Structure et Proprietes des Surfaces Solides, Colloque CNRS (Paris), 187, p.123, (1969).

31. M.Kotelitz, J.Oudar, Etude thermodynamique et structurale de l'adsorption dans le systeme or-soufre // Surf. Sci. 27 (1971).

32. M.Kotelitz, J.L.Domange, J.Oudar, Etude par la diffraction des electrons lents et la spectroscopie Auger de l'adsorption du soufre sur l'or // Surf. Sci. 34, 431-449 (1973).

33. M. Freemantle, Chemistry in action, Macmillan Education, London (1987).

34. K.V.Kostioukevich, B.A.Snopok, S.A..Zinio, Y.M.Shirshov, I.N.Kolesnikova, E.V.Lugovskoi, New opto-electronic system based on the surface plasmon resonance phenomenon: application to the concentration determination of DD-fragment of fibrinogen, in Opto-Contact: Workshop on Technology Transfers, Start-Up Opportunities, and Strategic Alliances - R.J.L.Corriveau, M.J.Soileu, M.Auger, Editors // Proceedings of SPIE 3414, pp. 290-301 (1998).

35. B.A.Snopok, K.V.Kostyukevych, O.V.Rengevych, Y.M.Shirshov, E.F.Venger, I.N.Kolesnikova, E.V.Lugovskoi, A Biosensor Approach to Probe the Structure and Function of the Adsorbed Proteins: Fibrinogen at the Gold Surface // Semiconductor Physics, Quantum Electronics \& Optoelectronics 1, pp. 121-134 (1998).

36. D.G.Myszka, Kinetic analysis of macromolecular interactions using surface plasmon resonance biosensors // Current Opinion in Biotechnology 8, pp. $50-57$ (1997).
37. O. V. Rengevich, Yu. M. Shirshov, Yu. V. Ushenin, A. G. Beketov, Separate determination of thickness and optical parameters by surface plasmon resonance: accuracy consideration // Semiconductor Physics, Quantum Electronics \& Optoelectronics 2(2), pp. 28-35 (1999).

38. A.B.Rubin, Biophysics, Vysshaia Shkola, Moskva (1987).

39. C.R.Cantor, P.R.Schimmel, Biophysical Chemistry, W.H.Freeman and Company (1980)

40. V.Frattali, R.F.Steiner, Separation and some properties of three inhibitors from commercial crude soybean trypsin inhibitor // Biochemistry 7(2), pp. 521-529 (1968).

41. M.Kunitz, Crystalline soybean trypsin inhibitor // J. Gen. Physiol. 30, pp. 291-310 (1947).

42. J.A. De Feijter, J.Benjamins and F.A.Veer // Biopolymers 17, p. 1759 (1978)

43. G.S.Retzinger, B.C.Cook, A.P.Deanglis, The binding of fibrinogen to surfaces and the identification of two distinct surface-bond species of the protein // J. Colloid Interface Sci. 168, pp. 514-521 (1994)

44. H.Fritz, E.Fink, M.Gebhardt, K.Hochstrasser, E.Werle // J. Physiol. Chem. 359, p. 933 (1969).

45. R.J.Green, J.Davies, M.C.Davies, C.J.Roberts and S.J.B.Tendler, Surface plasmon resonance for real time in situ analysis of protein adsorption to polymer surfaces // Biomaterials 18, pp. 405-413 (1997).

46. P.Schaaf, P.Dejardin and A.Schmitt, Reflectometry as a technique to study the adsorption of human fibrinogen at the silica/solution interface // Langmuir 3, pp. 1131-1135 (1987).

47. X.Baur, M.Pau, A.Czuppon, G.Fruhmann, Characterization of soybean allergens causing sensitization of occupationally exposed bakers // Allergy 51(5), pp. 326-30 (1996).

48. F. Vogtle, Supramolecular Chemistry, John Wiley \& Sons., London (1993).

49. Yu.Lvov, H.Moehwald, Protein Architecture: Interfacing Molecular Assemblies and Immobilization Biotechnology, Marcel Dekker, Inc.(1999).

50. Y. Kagava, Biomembrane, Vysshaia Shkola, Moskva (1985). 